\title{
La Seguridad en la Producción de Alimentos en la Granja - Resumen de Buenas Prácticas Agrícolas ${ }^{1}$
}

\author{
Federico G. Caro, Renee Goodrich Schneider, Keith R. Schneider, y Douglas L. Archer ${ }^{2}$
}

\section{Introducción}

Las Buenas Prácticas Agrícolas (BPA) y Buenas Prácticas de Manejo (BPM) cubren los procedimientos generales que los productores, empacadores y procesadores de frutas y verduras frescas deben seguir para garantizar la seguridad de sus productos. Las BPA son usadas antes de la cosecha (es decir, en el campo), mientras que las BPM se utilizan luego de la cosecha, incluyendo el embalaje y envío. En general, cuando decimos BPA incluimos todas las prácticas pre y post cosecha relacionadas con el manejo adecuado de productos frescos y mínimamente procesados tales como frutas y verduras.

El objetivo de este panfleto es revisar los principios generalmente reconocidos como BPA en la producción de frutas $\mathrm{y}$ verduras frescas, sobre todo a nivel de fincas o granjas. Otros panfletos de la Extensión Cooperativa de la Florida sobre la Inocuidad de los Alimentos cubren los principios individuales en detalle, con énfasis especial en los cultivos y el manejo apropiado de los mismos en la Florida.

\section{Son obligatorias las BPA?}

Desde el punto de vista legal las BPA se consideran recomendaciones y no son obligatorias, aunque es posible que los brotes recientes de enfermedades transmitidas por los alimentos puedan fomentar un cambio hacia algún tipo de requisito reglamentario obligatorio. Desde el punto de vista comercial, pueden existir requisitos de compra y programas específicos relacionados con las BPA que deben ser cumplidos y formalmente documentados por los productores y empacadores. Este tipo de requisitos es común en empresas grandes especialmente cuando los productos son exportados a otros países. Además, algunos estados, y algunos productos básicos que operan bajo órdenes de comercialización específicas, pueden tener requisitos específicos relacionados con las BPA o programas similares.

\section{Antecedentes}

En 1998, la Administración de Alimentos y Fármacos de los EE.UU. (FDA) publicó la Guía para Reducir al Mínimo el Riesgo Microbiano en Frutas y Hortalizas Frescas (1). El objetivo de este documento es el de advertir a los productores nacionales y extranjeros, empacadores, y transportistas de frutas y vegetales sobre los riesgos microbianos en los alimentos y aportar sugerencias para cada operación individual. La FDA indicó que estas normas son extensas y de carácter voluntario. Los temas detallados en la Guía forman la base de las BPA.

Al mismo tiempo, la Universidad de Cornell lanzó un programa importante llamado Programa de BPA Nacional

1. The English version of this document is FSHN06-01, Food Safety on the Farm - An Overview of Good Agricultural Practices. Este documento, FSHN06-01s, es uno de una serie de publicaciones del Food Science and Human Nutrition, Servicio de Extensión Cooperativa de la Florida, Instituto de Alimentos y Ciencias Agrícolas, Universidad de la Florida. (UF/IFAS). Fecha de primera publicación: May 2013. Visite nuestro sitio web EDIS en <http://edis.ifas.ufl. edu>.

2. Federico G. Caro, technician; Renee Goodrich Schneider, associate professor, and Keith R. Schneider, associate professor, Food Science and Human Nutrition Department; Douglas L. Archer, associate research dean, Florida Cooperative Extension Service, Institute of Food and Agricultural Sciences, University of Florida, Gainesville, FL 32611. 
con el objetivo de servir como la principal fuente de información universitaria y centro de intercambio de información sobre las BPA, su investigación y servicio de extensión (2). El sitio web de este programa es un recurso valioso con información sobre las BPA para los productores, empacadores y entrenadores. Este documento, $L a$ inocuidad de los Alimentos Empieza en el Campo - Guía del Agricultor, se puede encontrar en http://www.gaps.cornell. edu/FSBFEng.html. Este folleto está escrito en un lenguaje fácil de entender y está disponible en inglés y español.

En respuesta y reconocimiento de los crecientes problemas en la inocuidad alimentaria, la Ley de Modernización Para la Inocuidad de los Alimentos fue aprobada por el Congreso y firmada por el Presidente en Enero del 2011. La nueva ley exige a las empresas a implementar un programa de seguridad en la producción de alimentos que minimice significativamente los riesgos asociados con enfermedades transmitidas por alimentos contaminados. La Administración de Alimentos y Fármacos (FDA) también establecerá normas científicas para la producción segura de los productos frescos. A la luz de la nueva ley, los programas de BPA son cada vez más importantes. El objetivo de estos programas es estandarizar las BPA a nivel mundial y en todos los sectores a través de GLOBAL BPA y la Unión para la Iniciativa de Armonización de BPA $(3,4)$. La adopción de medidas inmediatas para implementar BPA beneficiará a las empresas y la inocuidad de los productos.

\section{Puntos acerca de las BPA}

Los siguientes puntos y comentarios se deberían considerar en cualquier programa integral sobre la inocuidad en la producción de alimentos. La lista a seguir se puede utilizar como una herramienta de evaluación preliminar para los operadores.

\section{Agua}

La calidad del agua utilizada impacta directamente el potencial de riesgo de contaminación de los productos frescos.

- Se debe inspeccionar el agua utilizada para el riego, para la mezcla de pesticidas y otros productos de aplicación foliar, el agua para la protección contra las heladas, el agua de procesamiento (por ejemplo su uso en canales de descarga, saneamiento y refrigeración de los productos) y para el saneamiento del equipo.

- El operador debe tener en cuenta el origen, la distribución y la calidad de toda el agua utilizada.

\section{El estiércol y los biosólidos municipales}

El estiércol y los biosólidos tratados adecuadamente pueden ser fertilizantes eficaces y seguros si se toman las debidas precauciones.

- Use tratamientos para reducir los patógenos en materiales orgánicos como el estiércol y otros. Los tratamientos pueden ser activos (por ejemplo, el compostaje) o pasivos (por ejemplo, el envejecimiento).

- Tenga en cuenta que el tratamiento y almacenamiento de estiércol cerca de los campos de cultivo aumenta el riesgo de contaminación.

- Considere factores como la pendiente, la lluvia y la posible infiltración de contaminantes en las áreas de producción de vegetales.

\section{La salud e higiene de los trabajadores}

Los empleados contagiados con una enfermedad infecciosa o con falta de higiene personal comprometen la sanidad de los productos frescos aumentando el riesgo de transmisión de enfermedades transmitidas por alimentos. Se sugiere:

- Capacitar a los empleados en buenas prácticas de higiene.

- Establecer un programa de capacitación sobre la salud e higiene. Incluya conceptos básicos, tales como las técnicas de lavado de manos y la importancia de usar el baño.

- Familiarizarse con los signos y síntomas típicos de enfermedades infecciosas.

- Proteger las heridas de inmediato si hay trabajadores con cortes o lesiones en partes del cuerpo que puedan entrar en contacto con productos frescos.

\section{Instalaciones sanitarias}

El manejo incorrecto de los desechos humanos y de otros tipos de contaminantes en el campo o la planta empacadora puede aumentar significativamente el riesgo de contaminación de los productos.

- Los baños deben estar correctamente ubicados.

- Los baños deben ser accesibles.

- Los servicios sanitarios y estaciones de lavado de manos deben tener los suministros requeridos.

- Todas las instalaciones deben mantenerse limpias.

\section{Sanidad en el campo}


Los productos frescos pueden ser contaminados por el contacto con el suelo, los fertilizantes, el agua, los trabajadores y los equipos de recolección durante las actividades de pre-cosecha y cosecha.

- Limpie los envases o recipientes de cosecha antes de su uso.

- Tenga cuidado de no contaminar ningún producto fresco que ya está lavado, enfriado o envasado.

- Utilice el equipo de cosecha y empaque de manera adecuada y manténgalo lo más limpio posible.

- Asigne la responsabilidad de mantener el equipo a la persona a cargo.

\section{Instalaciones de empaque}

Mantenga las instalaciones de empaque en buen estado para reducir la posibilidad de contaminación microbiana.

- Quite la mayor cantidad de suciedad posible afuera de las instalaciones de empaque.

- Limpie los contenedores o recipientes antes de utilizarlos. Deseche los envases dañados.

- Mantenga el equipo de empaque y las zonas de almacenamiento limpias.

- Guarde los contenedores vacíos para protegerlos contra la contaminación.

- Establezca y mantenga un programa de control de plagas.

\section{Transporte}

El transporte adecuado de los productos frescos ayuda a reducir la posibilidad de contaminación microbiana.

- La buena higiene y prácticas sanitarias adecuadas se deben utilizar durante la carga, la descarga y la inspección de los productos frescos.

- Inspeccione los vehículos de transporte y asegúrese que estén libres de olores, suciedad y escombros antes de la carga.

- Evite dejar el producto cosechado en el sol y mantenga una temperatura adecuada durante todo el proceso de transporte.

- Maneje los productos frescos tratando de minimizar el daño físico de los mismos.

\section{Monitoreo}

La capacidad para rastrear e identificar el origen de un producto es un componente importante de los programas de seguridad alimentaria. En virtud de la Ley de Bioterrorismo de 2002, esto es ahora obligatorio para cualquier empacador o transportista de frutas y verduras frescas (5).

- La documentación debe incluir el origen del producto, la fecha de cosecha, identificación de la finca y un registro de quién maneja el producto.

- El producto debe ser monitoreado desde la granja hasta los envasadores, distribuidores, transportistas, y los vendedores.

\section{Referencias}

1. Administración de Alimentos y Fármacos (FDA). 1998. Guía para Reducir al Mínimo el Riesgo Microbiano en Frutas y Verduras Frescas. Washington, DC: EE.UU. El Departamento de Salud y Servicios Humanos, la FDA. Disponible en: http://www.fda.gov/Food/GuidanceComplianceRegulatoryInformation/GuidanceDocuments/ ProduceandPlanProducts/UCM064574. Consultado el 13 de marzo 2011.

2. Cornell University 2002. Network De Buenas Prácticas Agrícolas Para la Educación y la Formación. Ithaca, NY: Cornell University Departamento de Ciencia de los Alimentos. Disponible en: http://www.gaps.cornell.edu/ indexhighspeed.html. Consultado el 13 de marzo 2011.

3. GLOBAL G.A.P. 2011. Aseguramiento Integrado de Fincas Versión 4. Colonia, Alemania: GLOBAL G.A.P. Disponible en: http://www.globalgap.org/cms/front_content.php?idcat=176. Consultado el 13 de marzo 2011.

4. Asociación unida de productos frescos. 2010. Iniciativa de Armonización de las BPA. Washington, DC: United Fresh Produce Association. Disponible en: http://www.unitedfresh.org/newsviews/gap_harmonization. Consultado el 13 de marzo 2011.

5. Administración de Alimentos y Fármacos (FDA). 2009. Ley Contra el Bioterrorismo de 2002. Disponible en: http://www.fda.gov/regulatoryinformation/legislation/ ucm148797.htm. Consultado el 01 de septiembre 2011. 